\title{
Optimization of Fuzzy Portfolio Considering Stock Returns and Downside Risk
}

\author{
Indah Simamora' ${ }^{1}$, Rahayu Sashanti ${ }^{2}$ \\ ${ }^{1,2}$ Department of Mathematics, University of North Sumatera, Indonesia
}

\begin{abstract}
First we must present two fuzzy portfolio selection models where the objective is to minimize the downside risk constrained so that a given expected return should be achieved. We assume that the rates of returns on securities are approximated as LR-fuzzy numbers of the same shape, and that the expected return and risk are evaluated by interval-valued means. We establish the relationship between those mean-interval definitions for a given fuzzy portfolio by using suitable ordering relations. And then we compare those with a given not fuzzy portfolio one. Finally, we can get the effect of not fuzzy portfolio under downside risk measures.
\end{abstract}

Keywords: Portfolio, Stock return, Fuzzy expected return, Downside risk.

\section{Introduction}

The portfolio selection problem deals with how to form a satisfying portfolio. It is difficult to decide which securities should be selected because of the existence of uncertainty on their returns. Our main objective is to obtain the optimal proportions for creating a portfolio which respects the investor's declared preferences. It is assumed that the investors wish to strike a balance between maximizing the return and minimizing the risk of their investment.

The first mathematical formulation of the problem of selecting a portfolio in the framework of risk-return trade-off was provided by Markowitz [4]. That author's theory of portfolio management combines probability theory and optimization theory to model the behaviour of the economic agents. In the classical portfolio selection, a probability distribution of the return on the assets is assumed to be known, the return is quantified by means of its expected value and the variance of the portfolio return is regarded as the risk of the investment. This classical Mean-Variance (MV) model is valid if the expected return is multivariatenormally distributed and the investor is averse to risk and always prefers lower risk.

Fuzzy Set Theory has been widely used to solve many practical problems, including financial risk management, since it allows us to describe and treat imprecise and uncertain elements present in a decision problem. Then the imperfect knowledge of the returns on the assets and the uncertainty involved in the behaviour of financial markets may also be introduced by means of fuzzy quantities and/or fuzzy constraints. Different elements can be fuzzified in the portfolio selection problem. In our approach, the uncertainty of the returns on the assets is modeled by means of fuzzy quantities, hence different definitions of the average of a fuzzy number can be used to evaluate both the expected return and the risk of a given portfolio.

Our goal is to present a fuzzy downside risk approach for managing portfolio selection problems in the framework of risk-return trade-off using interval-valued expectations.

Section 2 is devoted to describing the relationship between those two interval-value means for portfolios built using fuzzy returns which have been modelled with LR-fuzzy numbers of the same shape. The development of its corresponding fuzzy downside risk functions is given in Section 3. Then, in Section 3.1 and 3.2 we present the formulation of the fuzzy portfolio model and illustrate our approach to selecting the optimal portfolio using an example.

\section{Fuzzy Expected Return}

In a standard formulation of the portfolio selection, an investor chooses $\mathrm{xj}$, the proportion of a total investment fund devoted to asset $\mathrm{j}^{\text {th }}$ for $\mathrm{n}$ risky assets, so the portfolio may be denoted by $P(x)=\{x 1, \ldots, x n\}$. Let us assume that $\mathrm{u}_{j}$ (respectively $\mathrm{l}_{\mathrm{j}}$ ) represents the maximum (respectively minimum) amount of total fund which can be invested in asset $\mathrm{j}^{\text {th }}$, then the following budget equation holds: $\sum_{j}^{n}$, being $\quad l_{j} \leq x_{j} \leq u_{j}, j$ : The constraint imply that short selling of the securities is not allowed. Let us denote by / the fuzzy return on the asset $\mathrm{j}^{\text {th }}$ in the portfolio $\mathrm{P}(\mathrm{x})$, then its interval-valued mean [3] is defined:

$$
E\left(\widetilde{R}_{j}\right)=\left[E_{*}\left(\widetilde{R}_{j}\right), E^{*}\left(\widetilde{R}_{j}\right)\right]
$$

Where

$$
E_{*}\left(\tilde{R}_{j}\right)=\int_{0}^{1}\left(\inf \tilde{R}_{j \alpha}\right) d \alpha, E^{*}\left(\tilde{R}_{j}\right)=\int_{0}^{1}\left(\sup \tilde{R}_{j \alpha}\right) d \alpha \text { and inf } \tilde{R}_{j \alpha} \text { and } \sup \tilde{R}_{j \alpha}
$$

Denote respectively the left and right extreme points of the $\alpha$-level cut of for $\alpha \in(0,1]$. By using the definition of the interval-valued possibilitic mean in [2] for , we will have:

$$
M\left(\tilde{R}_{j}\right)=\left\lfloor M_{*}\left(\tilde{R}_{j}\right), M^{*}\left(\tilde{R}_{j}\right)\right\rfloor
$$

where $M_{*}\left(\tilde{R}_{j}\right)=2 \int_{0}^{1} \alpha\left(\inf \tilde{R}_{j \alpha}\right) d \alpha$ and $M^{*}\left(\tilde{R}_{j}\right)=2 \int_{0}^{1} \alpha\left(\sup \tilde{R}_{j \alpha}\right) d \alpha$

And for LR-fuzzy numbers with strictly decresing references functions, the following inclusion holds:

$$
M\left(\widetilde{R}_{j}\right) \subset E\left(\widetilde{R}_{j}\right) \text {. }
$$

With respect to the relationship between the above meaninterval definitions, we will establish that for a given portfolio $P(x)$ these expectations may be considered more or 


\section{International Journal of Science and Research (IJSR) \\ ISSN (Online): 2319-7064}

Index Copernicus Value (2013): 6.14 | Impact Factor (2015): 6.391

less optimistic measures of the expected return, depending on the magnitude of the right and left spreads of the LRfuzzy number which represents the fuzzy portfolio.

Let us denote the return on the $\mathrm{j}^{\text {th }}$ asset by $\widetilde{R_{j}}=\left(a_{l j}, a_{u j}\right.$ assuming that the left and right

reference functions are all of the same shape for $j=1, \ldots, n$ the following linear combination expresses the total fuzzy return on a portfolio $P(x)=\{x 1, \ldots, x n\}$ :

$$
\tilde{P}=\sum_{j=1}^{n} \tilde{R}_{j} x_{j}=\left(\sum_{j=1}^{n} a_{b} x_{j}, \sum_{j=1}^{n} a_{u j} x_{j}, \sum_{j=1}^{n} c_{j} x_{j}, \sum_{j=1}^{n} d_{j} x_{j}\right)_{L R}=\left(P_{l}(x), P_{u}(x), C(x), D(x)\right)_{L R}
$$

where $x \mathrm{j} \in \mathfrak{R}+$ represents the proportion of the jth asset in the composition of the portfolio. It is widely acknowledged that the interval-valued expectations and remain additive in the sense of the addition of fuzzy numbers [2, 3]. Let us consider a fuzzy portfolio whose $\mathrm{L}$ and $\mathrm{R}$ reference functions belong to the power family with the same parameter $\mathrm{p}$, that is $\mathrm{L}(\mathrm{x})=\mathrm{R}(\mathrm{x})=\max \left\{0,1-|x|^{p}\right\}, \mathrm{p}>0$. Since the power reference functions are continuous and strictly decreasing, it follows that the interval-valued possibilistic mean is a subset of the interval-valued probabilistic mean [2]. We can verify that the $\alpha$-level sets of have the

following form:

$$
[\tilde{P}]^{\alpha}=\left[\inf \tilde{P}_{\alpha}, \sup \tilde{P}_{\alpha}\right]=\left[P_{l}(x)-C(x)(1-\alpha)^{1 / p}, P_{u}(x)+D(x)(1-\alpha)^{1 / p}\right],
$$

for $\alpha \in[0,1]$, and it is easy to see that

$$
E_{*}(\tilde{P})=\int_{0}^{1}\left(\inf \tilde{P}_{\alpha}\right) d \alpha=P_{l}(x)-C(x) \frac{p}{p+1}
$$

therefore

$$
E(\tilde{P})=\left[P_{l}(x)-C(x) \frac{p}{p+1}, P_{u}(x)+D(x) \frac{p}{p+1}\right] .
$$

On the other hand, we can evaluate

$$
\begin{aligned}
& M^{*}(\tilde{P})=2 \int_{0}^{1} \alpha\left(\sup \tilde{P}_{\alpha}\right) d \alpha=P_{u}(x)+2 D(x) \int_{0}^{1} \alpha(1-\alpha)^{1 / p} d \alpha=P_{u}(x)+D(x) \frac{2 p^{2}}{(p+1)(2 p+1)} \\
& \text { so } \\
& M(\tilde{P})=\left[P_{l}(x)-C(x) \frac{2 p^{2}}{(p+1)(2 p+1)}, P_{u}(x)+D(x) \frac{2 p^{2}}{(p+1)(2 p+1)}\right] .
\end{aligned}
$$

Since we are interested in comparing the profit of a fuzzy portfolio and some ordering relations cannot explain the ranking between two partially or fully overlapping intervals, we will then study the relationship between these intervalvalued expectations by using properly ordered relations. Bermudez et. al [1] said two cases about midpoints:

$$
\text { a. If } \mathrm{C}(\mathrm{x})>\mathrm{D}(\mathrm{x}) \text {, it holds that } \quad E(\tilde{P}) \text {. When the fuzzy }
$$

portfolio is a symmetric fuzzy number, $\mathrm{C}(\mathrm{x})=\mathrm{D}(\mathrm{x})$, the midpoints coincide and the above ordering relation trivially holds.

b. If the right spread is the largest, $\mathrm{C}(\mathrm{x})<\mathrm{D}(\mathrm{x})$, we calculate the grade of acceptability of the premise $M\left(\tilde{P}^{\prime}\right.$,

according to Definition 3:

$$
A_{\prec}=\frac{(D(x)-C(x)) \frac{p}{2(p+1)(2 p+1)}}{P_{u}(x)-P_{l}(x)+(D(x)+C(x)) \frac{p(4 p+1)}{2(p+1)(2 p+1)}}<\frac{1}{4 p+1} .
$$

\section{Fuzzy Optimization Models}

The portfolio selection problem deals with finding an optimal investment strategy to form a satisfying portfolio, taking into account the balance between maximizing the return and minimizing the risk of the investment. We then need to specify the expected mean and risk of the portfolio. The basic model determines the portfolio which minimizes risk while a predetermined level of expected return is guaranteed, so the problem can be formulated as follows:

$$
\begin{array}{ll}
\text { Min } & f u z z y \operatorname{risk}(P(x)) \\
\text { s.t. } & f u z z y \operatorname{return}(P(x)) \geq \tilde{\rho} \\
& \sum_{j=1}^{n} x_{j}=1 \\
& l_{j} \leq x_{j} \leq u_{j}, \quad j=1, \ldots, n
\end{array}
$$

Different models coexist to select the best portfolio according to their respective objective functions in this fuzzy framework of risk-return trade-off. Let us note that fuzzy downside risk can be represented as follows:

$$
\widetilde{w}(\widetilde{P})=\sum_{j=1}^{n}\left[0, a_{u j}-a_{l j}+\frac{1}{2}\left(c_{j}+d_{j}\right)\right] x_{j}
$$

so we see that the above problem can be considered as a linear programming problem with interval coefficients, where the desired return is expressed as following:

$$
E(\widetilde{P})=\sum_{j=1}^{n}\left[a_{l j}-\frac{1}{2} c_{j}, a_{u j}+\frac{1}{2} d_{j}\right] x_{j}
$$

and $\rho \sim$ is an interval. For the possibilistic mean-interval values, a similar linear programming interval program appears.

\subsection{Defuzzification process}

An essential question connected with solving the problem of fuzzy portfolio selection is related to the defuzzification process. It consists of finding a scalar representative value of a fuzzy number and it can be done in many different ways. In order to obtain a crisp solution of the above fuzzy model, we need to choose a crisp objective function and a value for the left-hand side of the total return constraint. In this paper we have decided to minimize the fuzzy downside risk considered as a crisp objective and we have chosen to reflect the maximization of the expected return on the portfolio by means of a suitable crisp constraint. First we minimize the upper limit of the mean interval evaluated in Proposition 3 [1], which corresponds to the fuzzy downside risk, in order to ensure that the non-desired deviations to the fuzzy expected return are minimal. The objective function of the crisp model is then:

$$
F 1(x)=\sum_{j=1}^{n}\left(a_{w j}-a_{l j}+\frac{1}{2}\left(c_{j}+d_{j}\right)\right) x_{j} \text { or } F 2(x)=\sum_{j=1}^{n}\left(a_{u j}-a_{b j}+\frac{1}{3}\left(c_{j}+d_{j}\right)\right) x_{j} .
$$

Concerning the crisp modelling of the fuzzy quantity which represents the expected return, it seems reasonable to choose 


\section{International Journal of Science and Research (IJSR) \\ ISSN (Online): 2319-7064 \\ Index Copernicus Value (2013): 6.14 | Impact Factor (2015): 6.391}

the middle point of the corresponding intervalvalued mean, in such a way that if the left-width of the fuzzy number is greater than the right-width, the mean of the core is displaced to the left to reflect that larger dispersion, otherwise the displacement is to the right. Therefore, one crisp model associated with this defuzzification proposal of the expected fuzzy risk and return can be stated as:

$$
\begin{array}{ll}
\text { (P1) Min } & \sum_{j=1}^{n}\left(a_{u j}-a_{l j}+\frac{1}{2}\left(c_{j}+d_{j}\right)\right) x_{j} \\
\text { s.t. } & \sum_{j=1}^{n}\left(\frac{1}{2}\left(a_{u j}+a_{l j}\right)+\frac{1}{4}\left(d_{j}-c_{j}\right)\right) x_{j} \geq \rho \\
& \sum_{j=1}^{n} x_{j}=1 \\
& l_{j} \leq x_{j} \leq u_{j}, \quad j=1, \ldots, n
\end{array}
$$

where $\rho$ is the minimum expected return required by an investor. On the other hand, when the interval-valued possibilistic mean is used, the statement is the following:

$$
\begin{aligned}
\text { (P2) Min } & \sum_{j=1}^{n}\left(a_{u j}-a_{l j}+\frac{1}{3}\left(c_{j}+d_{j}\right)\right) x_{j} \\
\text { s.t. } & \sum_{j=1}^{n}\left(\frac{1}{2}\left(a_{u j}+a_{l j}\right)+\frac{1}{6}\left(d_{j}-c_{j}\right)\right) x_{j} \geq \rho \\
& \sum_{j=1}^{n} x_{j}=1 \\
& l_{j} \leq x_{j} \leq u_{j}, \quad j=1, \ldots, n
\end{aligned}
$$

Clearly, a direct comparison between the performance of the linear programs (P1) and (P2), provided by the approximation of the return and risk expectations by using $E(P \sim)$ and $M(P \sim)$, respectively, can be easily made.

\subsection{Numerical Example}

\subsubsection{Full description of the fuzzy portfolios}

We shall illustrate the above results by a simple example with three assets whose returns are the following trapezoidal fuzzy numbers:

$\tilde{R}_{1}=(-11 ; 71 ; 5 ; 100) \tilde{R}_{2}=(-9 ; 36 ; 5 ; 55) \tilde{R}_{3}=(-6 ; 29 ; 12 ; 85)$

Applying an algorithm for finding the exact optimal solution in the sense of maximizing a given utility score, the authors find the following portfolios:

$$
\begin{aligned}
& P_{1}(x)=\{0,124 ; 0,373 ; 0,503\}, \\
& P_{2}(x)=\{0,163 ; 0,837 ; 0\}, \\
& P_{3}(x)=\{0,103 ; 0 ; 0,897\}, \\
& P_{4}(x)=\{0 ; 0 ; 1\}
\end{aligned}
$$

So, we get:

$P_{l}(x)=a_{l 1} x_{1}+a_{l 2} x_{2}+a_{l 3} x_{3}$

$$
\begin{aligned}
& =(-11)(0,124)+(-9)(0,373)+(-6)(0,503) \\
& =-1,364-3,357-3,018 \\
& =-7,739 \\
P_{u}(x) & =a_{u 1} x_{1}+a_{u 2} x_{2}+a_{u 3} x_{3} \\
& =(71)(0,124)+(36)(0,373)+(29)(0,503) \\
& =8,804+13,428+14,587 \\
& =36,819 \\
C(x) & =C_{1} x_{1}+C_{2} x_{2}+C_{3} x_{3} \\
& =(5)(0,124)+(5)(0,373)+(12)(0,503) \\
& =0,62+1,865+6,036 \\
& =8,521 \\
D(x) & =D_{1} x_{1}+D_{2} x_{2}+D_{3} x_{3} \\
& =(100)(0,124)+(55)(0,373)+(85)(0,503) \\
& =12,4+20,515+42,755 \\
& =75,67
\end{aligned}
$$

If we give parameter $\mathrm{p}=1$ for $\mathrm{L}(\mathrm{x})$ and $\mathrm{R}(\mathrm{x})$, so for $\mathrm{P}_{1}(\mathrm{x})$, we get:

$$
\begin{aligned}
E\left(P_{1}\right) & =\left[P_{l}(x)-C(x) \frac{p}{p+1}, P_{u}(x)+D(x) \frac{p}{p+1}\right] \\
& =\left[-7,739-(8,521) \frac{1}{1+1}, 36,819+(75,67) \frac{1}{1+1}\right] \\
& =[-12,000,74,654] \\
M\left(P_{1}\right) & =\left[P_{l}(x)-C(x) \frac{2 p^{2}}{(p+1)(2 p+1)}, P_{u}(x)+D(x) \frac{2 p^{2}}{(p+1)(2 p+1)}\right] \\
& =\left[-7,738-(8,521) \frac{1}{3}, 36,819+(75,67) \frac{1}{3}\right] \\
& =[-10,579,62,042]
\end{aligned}
$$

Table 1: Full description of the fuzzy portfolios provided by Example

\begin{tabular}{ccccccc}
\hline & $P_{l}(x)$ & $P_{\mathrm{u}}(x)$ & $C(x)$ & $D(x)$ & $E(P i)$ & $M(P i)$ \\
\hline$P_{1}(x)$ & $-7,739$ & 36,819 & 8,521 & 75,670 & {$[-12,000,74,654]$} & {$[-10,579,62,042]$} \\
$P_{2}(x)$ & $-9,326$ & 41,705 & 5,000 & 62,335 & {$[-11,826,72,873]$} & {$[-10,993,62,483]$} \\
$P_{3}(x)$ & $-6,515$ & 33,326 & 11,279 & 86,545 & {$[-12,155,76,599]$} & {$[-10,275,62,174]$} \\
$\left.P_{4} x\right)$ & $-6,000$ & 29,000 & 12,000 & 85,000 & {$[-12,000,71,500]$} & {$[-10,000,57,333]$} \\
\hline
\end{tabular}

But the acceptability index allows us to generate the following ordering:

$$
E\left(P_{4}\right)<E\left(P_{2}\right)<E\left(P_{1}\right)<E\left(P_{3}\right)
$$

And suggests the selection of the maximizing alternative $\mathrm{P} 3(\mathrm{x})$, which is preferred to the other three intervals. In fact, we may say that thedecision maker is satisfied that $\mathrm{E}\left(\mathrm{P}_{1}\right)$ is inferior to $\mathrm{E}\left(\mathrm{P}_{3}\right)$ and his grade of satisfaction is 


\section{International Journal of Science and Research (IJSR) \\ ISSN (Online): 2319-7064 \\ Index Copernicus Value (2013): 6.14 | Impact Factor (2015): 6.391}

$$
\begin{aligned}
A \prec & =\frac{\frac{1}{12}(D(x)-C(x))}{P_{u}(x)-P_{l}(x)+\frac{5}{12}(D(x)-C(x))}<\frac{1}{5} \\
& =\frac{\frac{1}{12}(86,545-11,279)}{33,326+6,567+\frac{5}{12}(86,545+11,279)} \\
& =\frac{\frac{1}{12}(75,266)}{80,653}=0,077<\frac{1}{5}
\end{aligned}
$$

If $\mathrm{C}(\mathrm{x})<\mathrm{D}(\mathrm{x})$ and $\mathrm{M}(\mathrm{P})<\mathrm{E}(\mathrm{P})$ so acceptability grade interval $(0,1)$ proofed. And if $p>0$ (we use $p=1$ ) so the upper bound is $A \prec=0,2$. Then, if the interval-valued possibilistic mean is used, it should be more frequent to find infeasible instances of the optimization problem for a given $\rho$. This is the fact if we suppose that an investor wants to allocate one unit of wealth among the three assets with an expected return greater than or equal to $\rho=35$. For the upper bound $=0.4$ :

$$
\begin{aligned}
F_{1}(x)= & \left(\left(71-(-11)+\frac{1}{2}(5+100)\right) 0,268\right)+\left(\left(36-(-9)+\frac{1}{2}(5+55)\right) 0,332\right) \\
& +\left(\left(29-(-6)+\frac{1}{2}(12+85)\right) 0,4\right)=94,346 \\
F_{2}(x)= & \left(\left(71-(-11)+\frac{1}{3}(5+100)\right) 0,268\right)+\left(\left(36-(-9)+\frac{1}{3}(5+55)\right) 0,332\right) \\
& +\left(\left(29-(-6)+\frac{1}{3}(12+85)\right) 0,4\right)=80,08
\end{aligned}
$$

Table 2: Optimal portfolio selection obtained by solving (P1) with different upper bounds for the assets and $\rho=35$

\begin{tabular}{ccccccc}
\hline Upper Bound & 0,4 & 0,5 & 0,6 & 0,7 & 0,8 & 1 \\
\hline$x_{1}$ & 0,268 & 0,254 & 0,239 & 0,045 & 0,211 & 0,211 \\
$x_{2}$ & 0,332 & 0,246 & 0,161 & 0,255 & 0 & 0 \\
$x_{3}$ & 0,4 & 0,5 & 0,6 & 0,7 & 0,789 & 0,789 \\
$\sum x_{i}$ & 1 & 1 & 1 & 1 & 1 & 1 \\
\hline$m(E)$ & 35 & 35 & 35 & 35 & 35 & 35 \\
\hline$F_{1}(x)$ & 94,346 & 94,363 & 94,321 & 83,628 & 94,261 & 94,261 \\
\hline$F_{2}(x)$ & 80,08 & 79,556 & 78,9825 & 69,0521 & 77,9159 & 77,9159 \\
\hline & & & & & &
\end{tabular}

Table 2 shows the portfolios provided by solving the linear program (P1) under the same diversification conditions. The associated crisp values of the return and risk appear in the last rows. Since all the obtained interval-valued expectations are centred in the same value $\rho$, the main differences between the proposals are in the values of the risk provoked by the diversification conditions.

If we use not fuzzy optimization, $\mathrm{x}_{1}, \mathrm{x}_{2}, \mathrm{x}_{3}$ is gotten from selected asset, $\mathrm{P}_{3}(\mathrm{x})$. So we get $\mathrm{F}_{1}(\mathrm{x})$ and $\mathrm{F}_{2}(\mathrm{x})$ :

\begin{tabular}{ccccccc}
\hline Upper Bound & 0,9 & 0,9 & 0,9 & 0,9 & 0,9 & 0,9 \\
\hline$x_{1}$ & 0,103 & 0,103 & 0,103 & 0,103 & 0,103 & 0,103 \\
$x_{2}$ & 0 & 0 & 0 & 0 & 0 & 0 \\
$x_{3}$ & 0,897 & 0,897 & 0,897 & 0,897 & 0,897 & 0,897 \\
$\sum x_{i}$ & 1 & 1 & 1 & 1 & 1 & 1 \\
\hline$m(E)$ & 35 & 35 & 35 & 35 & 35 & 35 \\
\hline$F_{1}(x)$ & 88,168 & 88,168 & 88,168 & 88,168 & 88,168 & 88,168 \\
\hline$F_{2}(x)$ & 71,978 & 71,978 & 71,978 & 71,978 & 71,978 & 71,978 \\
\hline
\end{tabular}

So if we take not fuzzy optimization, we get only one portfolio selection for each $F_{1}(x)$ and $F_{2}(x)$ because of using single upper bound for all $\mathrm{F}_{1}(\mathrm{x})$ and $\mathrm{F}_{2}(\mathrm{x})$.

\subsubsection{The best combination of stocks (numerical results)}

This is the other example using downrisk method by varying the stock returns.
Indah and Rahayu desire a portfolio consisting of 5 stocks. What is the best combination of stocks to minimize risk for a given return?

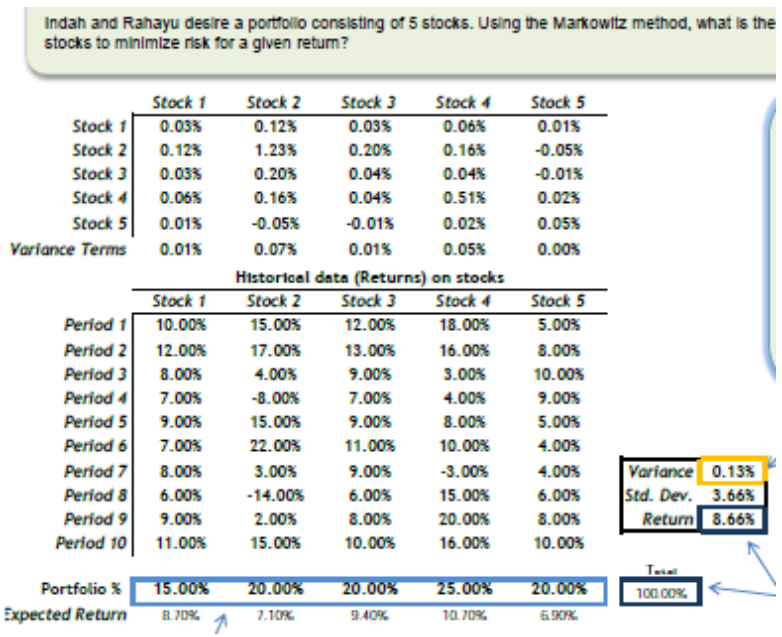

Using historical price data to compute estimates of stock returns, variances, and covariances is only a first step in investment planning. Stock returns, as well as variances and covariances, vary over time (fuzzy optimization). Investors often rely on security analysts to provide better estimates of these quantities for the future.

\section{Conclusion}

A number of portfolio selection models use the sample average to estimate the expected return on a given asset. In particular, the classical mean-variance model is a wellestablished method which provides good results. However, it is also well-known that the sample average is not always the best option for describing a data set and we believe that our fuzzy approach could be a good alternative in some of these situations.

Fuzzy methodology allows us to incorporate uncertainty into data bases and also to incorporate subjective characteristics into the models, which are basic aspects for establishing different estimations of risk and expected return.

Taking the uncertainty of returns on assets in a financial market as trapezoidal LR-fuzzy numbers, we generalize the mean semi-absolute deviation using both interval-valued probabilistic and possibilistic means. Then, based on this risk measure, we formulate two portfolio selection problems which can be solved using linear programming problems.

Clearly, a direct comparison between the performance of the linear programs (P1) and (P2) in fuzzy optimization model and in not fuzzy optimization is sum of portfolio selection. Not fuzzy optimization cause we get only one portfolio selection for each $F_{1}(x)$ and $F_{2}(x)$ because of using single upper bound and $\mathrm{x}_{1}, \mathrm{x}_{2}, \mathrm{x}_{3}$ from first selected asset, $\mathrm{P}_{3}(\mathrm{x})$ for all $\mathrm{F}_{1}(\mathrm{x})$ and $\mathrm{F}_{2}(\mathrm{x})$.

\section{References}

[1] Bermudez J. D., Segura J.V. and Vercher E., (2005), Fuzzy portofolio optimization under downside risk 


\section{International Journal of Science and Research (IJSR) \\ ISSN (Online): 2319-7064}

Index Copernicus Value (2013): 6.14 | Impact Factor (2015): 6.391

measures, Centro de Investigacion Operativa, Universidad Miguel Hernandez de Elche.

[2] C. Carlsson, R. Fullér, On possibilistic mean value and variance of fuzzy numbers, Fuzzy Sets and Systems 122 (2001) 315-326.

[3] D. Dubois, H. Prade, The mean value of a fuzzy number, Fuzzy Sets and Systems 24 (1987) 279-300.

[4]H. M. Markowitz, Portfolio selection: Efficient Diversification of Investments, John Wiley, New York, 1959. 